\title{
Design and rationale of the high-sensitivity Troponin T Rules Out Acute Cardiac Insufficiency Trial
}

This article was published in the following Dove Press journal:

Pragmatic and Observational Research

18 May 2017

Number of times this article has been viewed

\author{
Benton R Hunter ${ }^{\prime}$ \\ Sean P Collins ${ }^{2}$ \\ Gregory J Fermann ${ }^{3}$ \\ Phillip D Levy ${ }^{4}$ \\ Changyu Shen ${ }^{5}$ \\ Syed Imran Ayaz ${ }^{4}$ \\ Mette L Cole' \\ Karen F Miller ${ }^{2}$ \\ Adam A Soliman ${ }^{3}$ \\ Peter S Pang ${ }^{1,6}$
}

'Department of Emergency Medicine, Indiana University School of Medicine, Indianapolis, IN, ${ }^{2}$ Department of Emergency Medicine, Vanderbilt University Medical Center, Nashville, TN, ${ }^{3}$ Department of Emergency Medicine, University of Cincinnati, Cincinnati, $\mathrm{OH},{ }^{4}$ Department of Emergency Medicine, Wayne State University School of Medicine, Detroit, MI, ${ }^{5}$ Department of Biostatistics, Indiana University School of Medicine, ${ }^{6}$ Department of Indianapolis EMS, The Regenstrief Institute, IN, USA

\footnotetext{
Correspondence: Benton R Hunter Department of Emergency Medicine, Indiana University Health Methodist Hospital, I70I North Senate Avenue, Indianapolis, IN 46202, USA

$\mathrm{Tel}+\mathrm{I} 3174132318$

Email brhunter@iu.edu
}

Background: Acute heart failure (AHF) is a common presentation in the Emergency Department (ED), and most patients are admitted to the hospital. Identification of patients with AHF who have a low risk of adverse events and are suitable for discharge from the ED is difficult, and an objective tool would be useful.

Methods: The highly sensitive Troponin T Rules Out Acute Cardiac Insufficiency Trial (TACIT) will enroll ED patients being treated for AHF. Patients will undergo standard ED evaluation and treatment. High-sensitivity troponin T (hsTnT) will be drawn at the time of enrollment and 3 hours after the initial draw. The initial hsTnT draw will be no more than 3 hours after initiation of therapy for AHF (vasodilator, loop diuretic, noninvasive ventilation). Treating clinicians will be blinded to hsTnT results. We will assess whether hsTnT, as a single measurement or in series, can accurately predict patients at low risk of short-term adverse events.

Conclusion: TACIT will explore the value of hsTnT measurements in isolation, or in combination with other markers of disease severity, for the identification of ED patients with AHF who are at low risk of short-term adverse events.

Keywords: heart failure, high-sensitivity troponin, hospitalization

\section{Introduction}

Acute heart failure (AHF) is a major public health problem, accounting for over 1 million hospitalizations annually in the US. ${ }^{1}$ The vast majority of these admissions are through the Emergency Department (ED), ${ }^{1-3}$ where $85 \%$ of patients with AHF are hospitalized. ${ }^{3}$ These patients carry $\sim 4 \%$ risk for mortality during their hospitalization, and the risk of death approaches $12 \%$ within 30 days after discharge. ${ }^{4}$ Additionally, nearly $35 \%$ are dead or rehospitalized within 90 days of discharge. ${ }^{5}$ Consequently, ED physicians are hesitant to discharge patients with AHF and generally admit them to the hospital. This results in enormous morbidity as well as financial costs. ${ }^{1,5}$

Identifying a subgroup of ED patients with AHF where hospitalization can be avoided either by direct discharge or after a brief stay in an observation unit is a critical evidence gap..$^{6-8}$ The Agency for Health care Research and Policy Guidelines has suggested that up to $50 \%$ of AHF patients could be discharged from the ED or observed for a brief period. ${ }^{9}$ Unfortunately, defining those patients appropriate for discharge has proven difficult. Certain "high-risk" features, such as low blood pressure, high natriuretic peptide (NP), or cardiac-specific troponin concentrations, ${ }^{10-12}$ worsening renal function, and hyponatremia, are all associated with poor outcomes. ${ }^{13}$ However, the absence of these high-risk features does not guarantee a good outcome. Accordingly, there is no universally accepted AHF patient group considered "safe" to discharge 
from the ED. Certain risk stratification tools show promise, though further research is needed. The 13-item STRATIFY (Improving Heart Failure Risk Stratification in the ED) prediction too ${ }^{14}$ was prospectively derived from a cohort of over 1,000 ED patients with AHF. In the STRATIFY derivation study, $7 \%$ of enrolled patients experienced a cardiovascular (CV) event within 5 days and $12 \%$ experienced an event within 30 days. The model was able to risk-stratify patients for serious events with a c-statistic of 0.68 (95\% confidence interval: $0.63-0.74)$. The negative predictive values for identifying true low-risk patients was $100 \%$ at a $3 \%$ threshold for events, $96 \%$ at a 5\% threshold, and $93 \%$ at a $10 \%$ threshold. The authors concluded that $13 \%$ of their patients could have been discharged with a very low risk of CV events. Other risk stratification tools have been derived as well, but none has gained widespread adoption for disposition decision making. ${ }^{15,16}$ Identification of patients safe for ED discharge remains an unmet need.

While contemporary troponin assays have both diagnostic and prognostic value in the management of patients with AHF, next-generation high-sensitivity cardiac troponins (hsTn) can detect more minor degrees of myocardial injury. ${ }^{10-12}$ The ability to quantify lower amounts of released troponin, and thus identify patients without such elevations, may discriminate patients at very low risk for adverse $\mathrm{CV}$ events and death. ${ }^{17} \mathrm{~A}$ recent post hoc analysis from a therapeutic trial demonstrated the absence of CV deaths through 180 days in patients with low high-sensitivity cardiac troponin $\mathrm{T}$ (hsTnT) levels. ${ }^{18}$ This hypothesis-generating finding requires prospective external testing in a broader population of ED patients with AHF. Thus, we designed the high-sensitivity cardiac Troponin T Rules Out Acute Cardiac Insufficiency Trial (TACIT), a multicenter, prospective, observational pilot study. TACIT will test whether serial negative hsTnT measurements can identify low-risk AHF patients in the ED who are safe for discharge or brief observation. TACIT will provide pilot data sufficient to inform the design of a definitive trial to test this hypothesis. Our secondary objective will be to externally validate the STRATIFY risk score.

\section{Design}

TACIT is a pragmatic, multicenter, prospective, observational, cohort pilot trial to test the value of serial hsTnT measurements in order to identify ED patients with AHF who are at very low risk of adverse outcomes. At least 500 patients will be enrolled across five hospitals at four academic centers. All sites will have approval from their local ethics committee or institutional review board. All patients enrolled in TACIT will provide written informed consent.
Highly qualified research teams will lead the study at their respective sites after being trained by the study primary investigator and team.

\section{Study population}

Formal inclusion and exclusion criteria are listed in Table 1. Enrolled patients will be aged $\geq 21$ years and will be diagnosed with AHF by the treating emergency physician. Patients will have received therapy for AHF (diuretics, vasodilators, noninvasive ventilation, etc), and will have a blood pressure $>100 \mathrm{mmHg}$. In keeping with the pragmatic, "real-world" design, the diagnosis of AHF will be made clinically by a treating physician with enough confidence that treatment for AHF in being initiated. We chose pragmatic criteria to facilitate early enrollment of patients who were diagnosed with and treated for AHF in the ED. While the ED diagnosis may not always agree with the hospital discharge diagnosis, ${ }^{6}$ this is the population of interest and reflects "real-world" clinical practice. Enrollment must occur within 3 hours of first ED therapy for AHF. We chose 3 hours as a reasonable timeframe to begin consideration of ED discharge.

The study design targets low-risk patients, as high-risk patients are readily identified by established prognostic markers. Subsequently, we chose to exclude patients who would be considered too complex to discharge, even if both hsTnT were normal and other criteria suggested low risk. As such, patients with life expectancy $\leq 6$ months, active cancer, history of heart transplant or ventricular assist device, renal disease requiring dialysis, hypotension (systolic blood pressure $<100 \mathrm{mmHg}$ ), use of inotropes or vasopressors, fever $>101.5^{\circ} \mathrm{F}$, acute coronary syndrome (ACS) as the cause for AHF (based on the presence of typical signs or symptoms, or new electrocardiogram changes) or documented ACS within the previous 30 days, atrial fibrillation with heart rate $>130$, known substance abuse likely to make follow-up difficult, or involvement in any interventional trial are excluded. Inclusion and exclusion criteria are detailed in Table 1.

This study has been registered at ClinicalTrials.gov, identifier NCT02592135.

\section{Study protocol}

TACIT will enroll patients as a convenience sample, based on study personnel availability at each site. At minimum, this will be Monday through Friday from 8 am to $5 \mathrm{pm}$. For some sites, 24/7 enrollment is possible. Patients will be prescreened according to chief complaint using an Institutional Review Board-approved screening protocol of ED electronic health records. Patients will be aged $\geq 21$ years with complaints possibly attributable to AHF, such as dyspnea, shortness of 
Table I Eligibility criteria

\begin{tabular}{|c|c|}
\hline Inclusion criteria & Exclusion criteria \\
\hline I. Age $\geq 21$ years & I. Life expectancy $\leq 6$ months \\
\hline 2. Patient diagnosed with AHF by the treating physician & $\begin{array}{l}\text { 2. Shock of any kind or use or planned use of inotropes (dobutamine, } \\
\text { dopamine, milrinone) or vasopressors. Any form of vasodilator is allowed }\end{array}$ \\
\hline $\begin{array}{l}\text { 3. Patient has received IV loop diuretic or vasodilator therapy } \\
\text { (by any route) or NIV for AHF }\end{array}$ & 3. Fever $>101.5^{\circ} \mathrm{F}$ \\
\hline 4. Provide informed written consent & $\begin{array}{l}\text { 4. Presumed ACS as the primary reason for presentation or ACS within } 30 \\
\text { days. Patients with troponin release outside of ACS (Type } 2 \text { MI) may be } \\
\text { included }\end{array}$ \\
\hline 5. $\mathrm{SBP}>100 \mathrm{mmHg}(\mathrm{SBP}<100$ at any time is an exclusion) & 5. AF with RVR > $130 \mathrm{bpm}$ at any time requiring medical intervention \\
\hline \multirow[t]{7}{*}{ 6. Enrolled within 3 hours of first AHF therapy } & 6. History of transplant of any kind or VAD patient \\
\hline & 7. ESRD requiring dialysis \\
\hline & $\begin{array}{l}\text { 8. Involved in any investigational trial (observational study where no } \\
\text { intervention is allowed) }\end{array}$ \\
\hline & 9. Currently under treatment for cancer of any kind \\
\hline & $\begin{array}{l}\text { 10. Known history of active alcoholism or drug abuse that may lead to } \\
\text { noncompliance }\end{array}$ \\
\hline & I I. Participating in any other interventional therapeutic or device trial \\
\hline & $\begin{array}{l}\text { 12. Any patient whom the investigator deems would be difficult to obtain } \\
\text { follow-up }\end{array}$ \\
\hline
\end{tabular}

Abbreviations: ACS, acute coronary syndrome; AF, atrial fibrillation; AHF, acute heart failure; ESRD, end-stage renal disease; IV, intravenous; MI, myocardial infarction; NIV, noninvasive ventilation; RVR, rapid ventricular response; SBP, systolic blood pressure; VAD, ventricular assist device.

breath, chest pain, leg swelling or edema, or weight gain, will be screened for enrollment. Those who meet the inclusion and exclusion criteria will be approached for written informed consent by trained study personnel. A screening log will be kept of all patients screened for possible enrollment.

As part of ED standard practice, patients will have blood draws including electrolytes, troponin, NPs, and hematology studies. Standard practice also includes obtaining an electrocardiogram and chest X-ray. Test results will be recorded by the study personnel once the patient is enrolled. Additionally, detailed demographics, past medical history, and medication lists will be obtained. Objective physical exam findings such as vital signs, weight, temperature, and height will also be recorded. ED treatments, disposition, and diagnoses will be recorded. These data will be entered into a secure, protected, online case report form using REDCap. ${ }^{19}$

In addition to standard care, once enrolled, patients will undergo baseline ( 0 -hour) and 3-hour ( \pm 1 hour) blood draws for hsTnT, as well as N-terminal prohormone of brain natriuretic peptide. Once drawn, these samples will be frozen and stored for central processing. Clinicians will be blinded to the cardiac troponin $\mathrm{T}$ results. The 0-hour hsTnT blood draw must occur within 3 hours of the first AHF treatment (ie, loop diuretic or vasodilator or noninvasive ventilation) given in the ED.

Follow-up will occur 90 days after discharge, whether discharge occurred from the ED or after an inpatient admission. Discharge from the hospital will be counted as day 5 in the hospital or the actual day of discharge, whichever comes first. As the median length of stay is 4 days in the US, and our focus is on low-risk patients, we chose day 5 to minimize the confounding effect of very long length of stay patients. However, we will record overall length of stay to allow for additional analyses. Medical records will be reviewed for all patients. Vital status phone calls will occur at 30 and 90 days after discharge. Additionally, at least three attempts at phone contact will be attempted, and if unsuccessful, an additional two attempts to an alternate contact will be undertaken. Dates of all events will be recorded.

Follow-up was initially planned for 30 days only. However, after $20 \%$ of the planned enrollment, a review of demographics and outcomes was undertaken. This review was blinded to all hsTnT results. It was noted that the event rate was lower than expected, and the decision was made to follow patients to 90 days instead of only 30 . For patients enrolled prior to this protocol change, 30-day phone followup was completed as per the original protocol. Additionally, there will be a 90-day medical record review to assess for further events.

\section{Endpoints}

Table 2 describes the clinical endpoints that will be evaluated in TACIT. The primary endpoint will be a composite of 90-day all-cause mortality and rehospitalization, includ- 
Table 2 TACIT endpoints

\section{Primary outcome \\ Composite of death or rehospitalization, including repeat ED visit \\ Secondary outcome \\ STRATIFY risk score \\ Exploratory outcomes \\ The STRATIFY risk score comprises the following: \\ I. All-cause mortality \\ 2. Days alive and out of the hospital \\ 3. CV-specific rehospitalization and ED revisits \\ 4. Cardiopulmonary resuscitation \\ 5. Mechanical cardiac support \\ 6. Intubation or mechanical ventilation \\ 7. Emergent dialysis \\ 8. Percutaneous coronary intervention or coronary artery bypass grafting}

9. Acute coronary syndrome

Abbreviations: CV, cardiovascular; ED, emergency department; STRATIFY, Improving Heart Failure Risk Stratification in the ED; TACIT, Troponin T Rules Out Acute Cardiac Insufficiency Trial.

ing ED revisits. Secondary and exploratory endpoints will include days alive and out of the hospital, CV-specific rehospitalization and ED revisits, all-cause mortality, cardiopulmonary resuscitation, mechanical cardiac support, mechanical ventilation, emergent dialysis, ACS, and percutaneous coronary intervention/coronary artery bypass grafting. Each site will perform their own adjudication of events, blinded to hsTn results. For the endpoint of myocardial infarction, the diagnosis will be based on standard diagnostic algorithms requiring an elevation in (nonhighly sensitive) troponin above the 99th percentile with a typical rise and fall of the biomarker.

\section{Evaluation of the prognostic value of hsTnT}

The prognostic value of negative serial hsTnT values for the composite endpoint of 90-day mortality and rehospitalization will be evaluated using a variety of methods, given the observational, descriptive study design. We will test the 99th percentile and manufacturers-suggested cutoff (including gender-based cutpoints) as potential thresholds for abnormal. Additionally, we will perform an Area Under the Curve Receiver Operating Characteristic analysis to determine optimal thresholds for hsTn in relation to outcomes. First, we will examine the sensitivity, specificity, and the c-statistic for hsTnT. Second, we will examine the event rate within each quartile of the hsTnT distribution and test any trend using a Mantel-Haenszel test. Third, we will use logistic regression to examine the added prognostic value of hsTnT in addition to known prognostic factors such as age, gender, serum sodium, blood pressure, NP concentrations, and renal function. We will compute net reclassification indices and integrated discrimination improvement for this purpose.

\section{Sample size considerations}

Based on previously published literature, we estimated $\sim 25 \%$ of patients will meet the primary endpoint of death or rehospitalization by 30 days. This would require $\sim 400$ patients in order to achieve 100 events. With 100 events, we will be able to control the error in the sensitivity estimate within $\pm 10 \%$ and the error in the specificity estimate within $\pm 6 \%$. Given our enrollment criteria targeting low-risk patients, we estimated a sample size of 500 patients. Gender-specific percentiles of hsTn will be tested, and other subgroup analyses will be explored, given the design as a pilot study. These include, but are not limited to the subgroups such as age, gender, ejection fraction, systolic blood pressure, organ injury biomarkers, and NP levels. A blinded interim analysis will occur after 250 patients are enrolled.

\section{Discussion}

Patients presenting to the ED with AHF are admitted at an alarmingly high rate. ${ }^{3}$ Ironically, this conservative approach leads to admissions for patients in whom there is no proven inpatient treatment to improve outcomes or reduce mortality. Diuretics, vasodilators, and inotropes are used to treat patients with AHF, yet none have been proven effective in reducing mortality among these patients. Despite the high overall morbidity of this population, a subset of AHF patients likely carry a good short-term prognosis. ${ }^{14-16}$ Whether admission in these low-risk patients offers any alteration in prognosis is unclear. Many patients might be safely discharged with a low risk of short-term adverse events, ${ }^{9}$ sparing the significant costs and morbidity associated with hospitalizations. A decision tool to identify low-risk patients and increase physician confidence for safe discharge would be of great value. TACIT aims to evaluate the usefulness of hsTnT measurements, in isolation or in conjunction with other clinical variables, for identifying AHF patients in the $\mathrm{ED}$ who are at lower risk for adverse events. If hsTnT can, in isolation, accurately identify patients who are at low risk, it may obviate the need for more complex decision scores. A single simple marker to identify low-risk patients with AHF would fulfill an unmet need. While patients enrolled in TACIT will not be sent home based on hsTnT results, the results will inform the design of a larger multicenter, definitive trial. 


\section{Other ED-based AHF risk stratification scores}

Previous studies have attempted to accurately risk-stratify patients with AHF in the hopes of identifying a subset of patients safe for discharge either immediately or after a brief ED observation. Lee et al risk-stratified AHF patients in terms of 7-day mortality using large administrative datasets. ${ }^{16}$ The authors randomly selected over 12,000 charts from 125 hospitals in Ontario, Canada for detailed abstraction. They included both admitted and discharged patients with AHF in the ED. Their final model included ten variables. They identified $25 \%$ of patients who had a $<0.5 \%$ risk of death at 7 days. However, this dataset did not include adverse events other than mortality, or outcomes beyond 7 days, which may not be long enough to give clinicians confidence to discharge patients. In addition, Canadian hospitals tend to have a higher ED discharge rate than US hospitals, potentially confounding comparisons.

In a validation of the AHF Index, Hsieh et al identified $19 \%$ of 8,384 AHF patients admitted to Pennsylvania hospitals as low risk. Among these low-risk patients, $2.4 \%$ had a serious in-hospital complication or died before hospital discharge, and 30-day mortality was $2.9 \% .{ }^{15}$ Results such as these have not given clinicians the confidence to adopt any current tools as disposition aids, and no stratification system has yet attained widespread adoption.

Among risk stratification studies, the largest prospective trial performed in an ED setting was the STRATIFY study. ${ }^{14}$ TACIT will attempt to validate these findings in a new population of prospectively enrolled ED patients with AHF.

A common theme among all of these risk stratification scores $^{14-16}$ : elevated troponin is highly predictive of both death and other serious $\mathrm{CV}$ events. Troponin is arguably the most predictive single laboratory test available for prognosis in patients with cardiac disease, and even in many patients with noncardiac pathology. Given the established prognostic value of troponin across a wide variety of conditions, including heart failure, we hypothesize that hsTn measurements will add significant value to current risk stratification.

\section{Limitations}

There are several important limitations to the TACIT pilot study. Foremost, it is observational in nature, and thus, we cannot use the findings to establish a causal relationship between use of hsTnT and patient outcomes. Further, we rely on physician assessment for the diagnosis of AHF. While we expect that there may be cases misclassified as AHF, our design allows a pragmatic approach to assessment of patients seen in the ED and thought to be suffering from AHF. This design will be important for real-world application of hsTn as a marker of low risk. Additionally, participating centers will be enrolling patients as a convenience sample. There may be some differences between patients with AHF presenting during "off hours" as opposed to those that we will capture during the day on weekdays. Because TACIT is being carried out at four academic referral centers, results in general may not be applicable to settings that differ in demographic, socioeconomic, or other baseline characteristics. Our study is also likely to be underpowered for outcomes other than our composite endpoint of mortality and rehospitalization. Rehospitalization itself is subject to factors that make it less objective than mortality, such as the risk tolerance of physicians and the preferences of each patient concerning their own dispositions. Despite this, rehospitalization is a common endpoint in AHF literature and carries importance to patients, clinicians, and payors.

\section{Conclusion}

The TACIT study will test the hypothesis that hsTnT measurements, either at presentation or serially, will provide clinically important prognostic information in patients with AHF who are not otherwise at high risk for adverse outcomes. Specifically, we hope to derive a decision instrument utilizing hsTnT in conjunction with other markers of disease severity that identifies patients with AHF who are at very low risk of 90-day adverse events.

\section{Acknowledgments}

TACIT is supported by an independent investigator grant from Roche pharmaceuticals. Research support: Roche, Novartis, PCORI. Research support: NIH, PCORI, Novartis, Roche, Trinity. Research support: Roche, Novartis, PCORI (FC14-1409-21656), NIH/NIMHD, NIH/NHLBI. Research support: Novartis, Siemens, Radiometer, Roche, PCORI, NIH, Speakers Bureau for Janssen.

\section{Disclosure}

Mette Cole receives research support from Novartis and Roche Diagnostics. Karen Miller receives research support from NIH, PCORI, Novartis, Roche, and Trinity Biotech.

Peter S Pang is or has been in the last 1 year a consultant for BMS, Janssen, Medtronic, Novartis, Trevena, scPharmaceuticals, Roche Diagnostics, and Relypsa. Sean P Collins is a consultant for Trevena, Cardiorentis, Novartis, Siemens, and Cardioxyl. Phillip Levy is a consultant for Janssen, Medtronic, The Medicines Company, Novartis, Trevena, 
Cardiorentis, Siemens, Roche Diagnostics, ZS Pharma, and Apex Innovations. Gregory J Fermann is a consultant for Pfizer and Janssen. The other authors report no conflicts of interest in this work.

\section{References}

1. Go AS, Mozaffarian D, Roger VL, et al. Heart disease and stroke statistics--2014 update: a report from the American Heart Association. Circulation. 2014;129:e28-e292.

2. Fonarow GC, Abraham WT, Cannon CP, Gheorghiade M, SacknerBernstein JD, Udelson JE. Role of beta-blocker therapy in the post-myocardial infarction patient with and without left ventricular dysfunction. Rev Cardiovasc Med. 2003;4(Suppl 3):S54-59.

3. Storrow AB, Jenkins CA, Self WH, et al. The burden of acute heart failure on U.S. emergency departments. JACC Heart Fail. 2014;2(3):269-277.

4. Adams KF Jr, Fonarow GC, Emerman CL, et al. Characteristics and outcomes of patients hospitalized for heart failure in the United States: rationale, design, and preliminary observations from the first 100,000 cases in the Acute Decompensated Heart Failure National Registry (ADHERE). Am Heart J. 2005;149(2):209-216.

5. Jencks SF, Williams MV, Coleman EA. Rehospitalizations among patients in the Medicare fee-for-service program. $N$ Engl J Med. 2009;360(14):1418-1428.

6. Collins SP, Lindsell CJ, Naftilan AJ, et al. Low-risk acute heart failure patients: external validation of the Society of Chest Pain Center's recommendations. Crit Pathw Cardiol. 2009;8(3):99-103.

7. Collins SP, Pang PS, Fonarow GC, Yancy CW, Bonow RO, Gheorghiade M. Is hospital admission for heart failure really necessary?: the role of the emergency department and observation unit in preventing hospitalization and rehospitalization. J Am Coll Cardiol. 2013;61(2):121-126.

8. Collins SP, Schauer DP, Gupta A, Brunner H, Storrow AB, Eckman $\mathrm{MH}$. Cost-effectiveness analysis of ED decision making in patients with non-high-risk heart failure. Am J Emerg Med. 2009;27(3): 293-302.
9. Graff L, Orledge J, Radford MJ, Wang Y, Petrillo M, Maag R. Correlation of the Agency for Health Care Policy and Research congestive heart failure admission guideline with mortality: peer review organization voluntary hospital association initiative to decrease events (PROVIDE) for congestive heart failure. Ann Emerg Med. 1999;34(4 Pt 1):429-437.

10. Mueller C, Giannitsis E, Christ M, et al. Multicenter evaluation of a 0-hour/1-hour algorithm in the diagnosis of myocardial infarction with high-sensitivity cardiac troponin T. Ann Emerg Med. 2016;68(1):76-87.

11. Rubini Gimenez M, Hoeller R, Reichlin T, et al. Rapid rule out of acute myocardial infarction using undetectable levels of high-sensitivity cardiac troponin. Int J Cardiol. 2013;168(4):3896-3901.

12. Zhelev Z, Hyde C, Youngman E, et al. Diagnostic accuracy of single baseline measurement of Elecsys Troponin T high-sensitive assay for diagnosis of acute myocardial infarction in emergency department: systematic review and meta-analysis. BMJ. 2015;350:h15.

13. Gheorghiade M, Pang PS. Acute heart failure syndromes. J Am Coll Cardiol. 2009;53(7):557-573.

14. Collins SP, Jenkins CA, Harrell FE Jr, et al. Identification of emergency department patients with acute heart failure at low risk for 30-day adverse events: the STRATIFY decision tool. JACC Heart Fail. 2015;3(10):737-747.

15. Hsieh M, Auble TE, Yealy DM. Validation of the acute heart failure index. Ann Emerg Med. 2008;51(1):37-44.

16. Lee DS, Stitt A, Austin PC, et al. Prediction of heart failure mortality in emergent care: a cohort study. Ann Intern Med. 2012;156(11):767-775, W-261, W-262.

17. Pang PS, Schuur JD. Emergency departments, acute heart failure, and admissions: one size does not fit all. JACC Heart Fail. 2014;2(3):278-280.

18. Pang PS, Teerlink JR, Voors AA, et al. Use of high-sensitivity troponin $\mathrm{T}$ to identify patients with acute heart failure at lower risk for adverse outcomes: an exploratory analysis from the RELAX-AHF trial. JACC Heart Fail. 2016;4(7):591-599.

19. Harris PA, Taylor R, Thielke R, Payne J, Gonzalez N, Conde JG. Research electronic data capture (REDCap)--a metadata-driven methodology and workflow process for providing translational research informatics support. J Biomed Inform. 2009;42(2):377-381.
Pragmatic and Observational Research

\section{Publish your work in this journal}

Pragmatic and Observational Research is an international, peer-reviewed, open access journal that publishes data from studies designed to reflect more closely medical interventions in real-world clinical practice compared with classical randomized controlled trials (RCTs). The manuscript management system is completely online and includes a very quick and fair peer-review

\section{Dovepress}

system. Visit http://www.dovepress.com/testimonials.php to read real quotes from published authors. 